\title{
Electrifying quantum dots for lasers
}

\section{Compositional grading of colloidal quantum dots enables electrically driven amplification of light, bringing electrically driven lasers from these materials very close}

\section{Ifor Samuel}

Lasers are now found throughout everyday life in DVD players, pointers, scanners and printers as well as in a huge range of scientific and commercial applications. They are special and important light sources because the light they emit is "coherent", which means that their emission is normally in a fine beam of well-defined wavelength that can be tightly focussed. Advances in lasers are closely related to advances in materials. Writing in Nature Materials, Jaehoon Lim, Young-Shin Park and Victor Klimov ${ }^{1}$ show that colloidal semiconductor nanoparticles (quantum dots) can amplify light when they are excited electrically. The results are expected to lead soon to the demonstration of electrically pumped lasing from this class of materials.

Quantum dots are tiny particles of semiconductors - typically with few nanometre dimensions. Colloidal quantum dots (CQDs) are made by simple chemical procedures in solution, in contrast to epitaxially grown quantum dots that need an expensive and complicated high vacuum system for their growth. The band gap of CQDs can be tuned by adjusting the synthesis to change their size, or (for larger changes) by changing the material. They can give efficient light emission, which coupled with their tuneability and simple fabrication from solution means they are used as colour converters in displays and for labelling biological and medical samples in microscopy. There has been considerable recent progress in light-emitting diodes made from CQDs ${ }^{2}$, and the demonstration of optically pumped lasing from these materials ${ }^{3,4}$.

There are two key components of a laser - a gain medium which can amplify light and a resonator that forces light to propagate backwards and forwards through the gain medium getting amplified to high intensity. The light amplification process happens by a process called stimulated emission in which one photon stimulates the emission of another photon with the same phase, wavelength and direction, leading to many photons with the same properties and hence coherence ${ }^{5}$ (Fig. 1a). In order to amplify light, there must be a population inversion, which means that more atoms/molecules/dots must be in an excited state than an unexcited state. So far electrically pumped lasing, where population inversion is achieved by passing a current through the gain medium, has proved very challenging for CQDs and other solution-processed materials such as organic semiconductors. A serious problem in both classes of material is that lasers require very high excitation densities to achieve population inversion, and this is 
difficult to deliver electrically without overheating (and damaging) the sample ${ }^{6}$. Another problem for CQDs is that, at the very high densities required to give gain, there is a rapid decay pathway called Auger recombination. In Auger recombination, an exciton transfers its energy to a nearby charge carrier, instead of emitting light. This has prevented sustained lasing in these materials because when they are excited towards a density high enough for lasing, the excitations decay by the non-radiative Auger process instead of by lasing.

Lim, Park and Klimov have made progress on both these issues. In particular they build on recent work ${ }^{7}$ ${ }^{8}$ to show that a shell around the quantum dot with graded composition can greatly reduce Auger recombination (Fig. 1b). This enables higher occupancy of the quantum dots, and an optical gain lifetime 4-5 times larger than for large quantum dots without the continuous grading. These features are very helpful for lasing, which can be readily achieved under optical excitation. The remaining - and considerable - challenge is to achieve the same high density under electrical excitation. This is because high current densities lead to heating that can easily destroy a device. The researchers used a device architecture with an insulating spacer that laterally limits the contact area between the CQD layer and the charge injection layers, thereby limiting the device area (Fig. 1C). Smaller devices have also proved helpful in making high brightness organic light-emitting diodes ${ }^{9}$. The advantage of this architecture is likely to be that it allows heat to diffuse laterally from the active area as well as vertically. It enables current densities of up to $18 \mathrm{~A} / \mathrm{cm}^{2}$ to be passed through the device at which point the average quantum dot occupancy is 3.2. This leads to the most important result in the paper which is the direct demonstration of optical amplification (gain) - when sufficient current is passed through the device, light incident on it is amplified.

The achievement of electrically pumped gain is a substantial advance and very nearly gives an electrically pumped laser. The next step will be to incorporate a device of this type into a resonator that applies feedback and so progress from the electrically pumped amplifier they have made to an electrically pumped laser. In principle, this achievement may be more accessible compared with the very difficult task of realising the electrically pumped amplifier. Further development of the work would be to make other colours of amplifier or laser. This initial work is an excellent proof of principle - but in the red region of the spectrum where very good diode lasers already exist. There is an opportunity to use the tuneability of band gap to shift the CQD laser emission in the green, a more challenging spectral range for current technologies, though attention will need to be paid to keep Auger recombination under control as the band gap is tuned. Moreover, the current proof of principle material contains cadmium so future work would naturally include developing non-toxic quantum dots that have similar properties - hopefully, the continuous grading approach will also work to suppress Auger recombination in these materials. As well as providing an imminent route to electrically pumped CQD lasers, the work also opens the way to exceptionally high brightness quantum dot LEDs that may see applications in displays and lighting. 
Ifor Samuel is at the Organic Semiconductor Centre, SUPA, School of Physics and Astronomy, University of St Andrews, St Andrews, U.K.

e-mail:idws@st-andrews.ac.uk

\section{References}

1. J. Lim, Y-S. Park, Klimov V. Optical gain in colloidal quantum dots achieved with direct-current electrical pumping. Nature Materials

2. Shirasaki Y, Supran GJ, Bawendi MG, Bulović V. Emergence of colloidal quantum-dot light-emitting technologies. Nature Photonics 2013, 7: 13-23.

3. Fan F, Voznyy O, Sabatini R, Bicanic K, Adachi M, McBride J, et al. Continuous-wave lasing in colloidal quantum dot solids enabled by facet-selective epitaxy. Nature Communications April 2017, 544: 75-79.

4. Pietryga J, Park Y, Lim J, Fidler A, Bae W, Brovelli S, et al. Spectroscopic and Device Aspects of Nanocrystal Quantum Dots. Chemical Reviews 2016, 116: 10513-10622.

5. Svelto O. Principles of Lasers. Springer 2010.

6. Samuel IDW, Namdas EB, Turnbull GA. How to recognize lasing. Nature Photonics 2009, 3: 546-549.

7. Nasilowski M, Spinicelli P, Patriarche G, Dubertret B. Gradient CdSe/CdS Quantum Dots with Room Temperature Biexciton Unity Quantum Yield. Nano Letters 2015, 15: 3953-3958.

8. Bae WK, Padilha LA, Park Y-S, McDaniel H, Robel I, Pietryga JM, et al. Controlled Alloying of the Core-Shell Interface in CdSe/CdS Quantum Dots for Suppression of Auger Recombination. ACS Nano 2013, 7: 3411-3419.

9. Matsushima T, Adachi C. High-current Injection and Transport on Order of kA/cm2 in Organic Light-emitting Diodes Having Mixed Organic/Organic Heterojunction Interfaces. Japanese Journal of Applied Physics 2007, 46: L861-L863. 

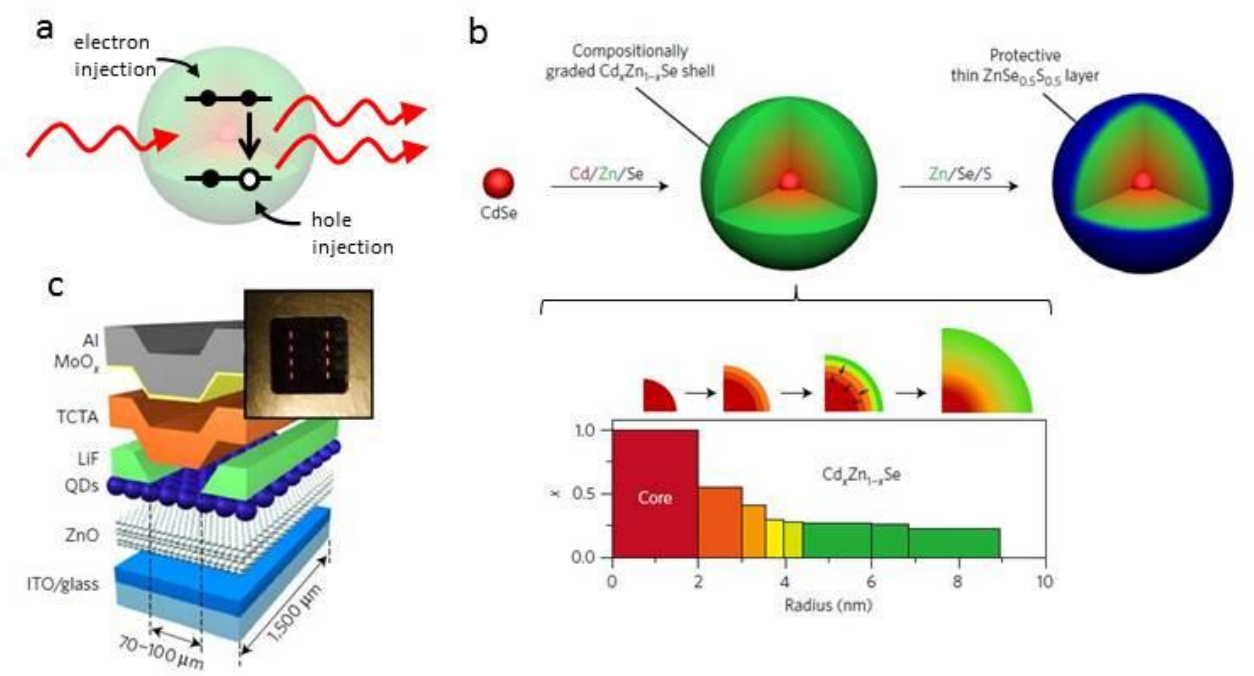

Figure 1. Compositionally graded quantum dots for electrically driven amplification of light. a. Schematic of a quantum dot with population inversion. A photon (red arrow) incident from the left stimulates the emission (vertical black arrow) of another photon with the same phase, wavelength and direction. The quantum dot is excited electrically by injecting electrons into the conduction band (upper line) and holes into the valence band (lower line). b. Compositionally graded quantum dots: a $2 \mathrm{~nm}$ radius CdSe core is surrounded by a $7 \mathrm{~nm} \mathrm{Cd} \mathrm{Zn}_{1-\mathrm{x}} \mathrm{Se}$ shell and a final $<1 \mathrm{~nm} \mathrm{ZnSe}_{0.5} \mathrm{~S}_{0.5}$ protective layer. $\mathrm{Zn}$ replaces $\mathrm{Cd}$ as distance from the centre increases. c. The device architecture used. Electrons are injected from the bottom through the indium tin oxide (ITO) and the $\mathrm{ZnO}$ layers, and holes from the top through the metal contacts and the tris(4-carbazoyl-9-ylphenyl)mine (TCTA) hole transport layer. They move under the applied field and recombine in the quantum dot (QD) layer. An insulating LiF layer is used so that current only flows through a narrow region, which enables higher current densities to be reached without damaging the device. 